\title{
Avaliação da atenção primária a saúde prestada à população infantil na perspectiva dos cuidadores
}

\author{
Evaluation of primary health care provided to the child population from the perspective of \\ caregivers
}
Evaluacion de la atencion primaria de salud prestada a la poblacion infantil desde la perspectiva de los cuidadores

Thaís Rodrigues Bezerra Nunes ${ }^{1 *}$, Fernando Rodrigues Peixoto Quaresma ${ }^{1}$, Soraya Rodrigues Dodero', Laisa Vaz dos Santos ${ }^{1}$.

\section{RESUMO}

Objetivo: Avaliar os atributos da Atenção Primaria à Saúde do ponto de vista dos usuários, em relação à saúde prestada à população infantil além de mensurar a efetividade dos atributos da APS. Métodos: Estudo transversal, descritivo, de abordagem quantitativa realizado com 131 pais/cuidadores de crianças de 0 a 2 anos cadastradas no Centro de Saúde e Comunidade 403 norte localizado em Palmas - TO. A coleta de dados foi realizada por meio de uma ferramenta para avaliação da atenção primária à saúde (PCA-Tool) versão infantil. Resultados: Os participantes avaliaram os atributos com escore geral alto, exceto os itens acessibilidade e orientação comunitária que tiveram baixo valor atribuído. Conclusão: Apesar dos serviços à saúde infantil terem sido bem avaliados de uma maneira geral, destacando-se integralidade e coordenação do cuidado, ainda há muito que se melhorar sobretudo a acessibilidade e orientação comunitária para consolidar a Atenção Primária à Saúde de forma ainda mais satisfatória.

Palavras-chave: Atenção primária à saúde, Saúde infantil, Avaliação em saúde.

\begin{abstract}
Objective: To evaluate the attributes of Primary Health Care from the point of view of users, in relation to the health provided to the infant population, in addition to measuring the effectiveness of the attributes of Primary Health Care. Method: A cross-sectional, descriptive, quantitative approach study conducted with 131 parents/caregivers of children from 0 to 2 years of age registered at the 403 North Health and Community Center located in Palmas - TO. Data were collected through a questionnaire used as a tool for the evaluation of primary health care (PCA-Tool) children's version. Results: Participants evaluated the attributes with high overall score, except for the items accessibility and community orientation that had low value attributed in the evaluation. Conclusion: Although child health services have been well evaluated in general, highlighting integrality and coordination of care, there is still a lot to improve especially accessibility and community guidance to consolidate Primary Health Care even more satisfactorily.
\end{abstract}

Keywords: Primary health care, Child health, Health evaluation.

${ }^{1}$ Fundação Escola de Saúde Pública de Palmas, Palmas - TO.

*E-mail: thaisrbnunes@hotmail.com

SUBMETIDO EM: 9/2020

ACEITO EM: 10/2020

PUBLICADO EM: 2/2021 


\section{RESUMEN}

Objetivo: Evaluar los atributos de la Atención Primaria de Salud desde el punto de vista de los usuarios, en relación con la salud brindada a la poblaciòn infantil, además de medir la efectividad de los atributos de la APS. Metodos: Un estudio transversal, descriptivo y cuantitativo realizado con 131 padres/cuidadores de niños de 0 a 2 años de edad registrados en el 403 North Health and Community Center ubicado en Palmas - TO. Los datos se reunieron mediante un cuestionario utilizado como instrumento para la evaluación de la versión infantil de la atención primaria de salud (PCA-Tool). Resultados: Los participantes evaluaron los atributos con una alta puntuación general, excepto los artículos de accesibilidad y orientación a la comunidad que tuvieron un bajo valor atribuido en la evaluación. Conclusión: Si bien los servicios de salud infantil han sido bien evaluados en general, destacando la integralidad y coordinación de la atención, aún queda mucho por mejorar especialmente la accesibilidad y la orientación comunitaria para consolidar la Atención Primaria de Salud aún más satisfactoriamente.

Palabras clave: Atención primaria de salud, Salud infantil, Evaluación de la salud.

\section{INTRODUÇÃO}

O conceito de Atenção Primária à Saúde (APS) dentro de um sistema de organização de serviço de saúde foi considerado pela primeira vez pelo Relatório de Dawson. Neste relatório, distinguiam-se três níveis principais de serviços da saúde: centros de saúde primários, centros de saúde secundários e hospitais-escola com funções interligadas (PORTELA GZ, 2016).

A Declaração de Alma-Ata foi outro importante marco para Atenção Primária à Saúde e reforçou o direito universal a saúde, tendo como base os cuidados primários de saúde. Em 2018, foi assinada a Declaração de Astana, reafirmando que havia sido colocado como prioridade na Declaração de Alma-Ata (GIOVANELLA L, et al.,2019).

Desde a criação do Sistema Único de Saúde (SUS) já haviam iniciativas em relação APS, porém, só se consolidou em 1990 com a municipalização do SUS, com maior estruturação da APS no intuito de garantir a descentralização e regionalização da saúde, onde os municípios se tornariam responsáveis pela saúde, por meio de normatizações e financiamento por parte do Ministério da Saúde (PORTELA GZ, 2016).

A Estratégia Saúde da Família (ESF) foi implantada no Brasil em 1994 como alternativa para reorientação do sistema de saúde, transgredindo do modelo assistencial para contemplar os princípios do SUS. É hoje a principal estratégia para consolidar a APS usado no país (BRASIL, 2011). Um dos principais pontos na construção desse modelo de atenção é a humanização que é norteada pelo acolhimento nos serviços de saúde (RIBEIRO LCC, et al., 2010).

A Unidade Básica de Saúde (UBS) recebe diariamente problemas e situações variadas que exigem condução de forma resolutiva demandando ações articuladas e por vezes intersetoriais (FREITAS TCC, et al., 2020). Levando em consideração que os problemas de saúde se distribuem de forma heterogênea em uma determinada população, surge a necessidade da criação de Redes de Atenção à Saúde (RAS), em que diversos pontos de Atenção à Saúde em variados níveis de complexidade estejam interligados. A instituição das RAS inclui diferentes atores com responsabilização coletiva e é vista como uma tentativa de superar um grande desafio do SUS: a fragmentação de ações e do cuidado (PEITER CC, et al., 2019).

Starfield B (2012) defende que, para uma APS bem definida, alguns atributos devem estar presentes, tornando-os atributos essenciais: atenção ao primeiro contato, longitudinalidade, integralidade e coordenação. A orientação familiar, orientação comunitária e a competência cultural entram como atributos derivados. Sendo assim, a efetividade da APS está relacionada ao seu grau de estruturação baseada nesses atributos (BRANQUINHO ID e LANZA FM, 2018). 
A APS é o espaço potencial para a promoção da saúde infantil no âmbito sanitário devido a abordagem integral do binômio saúde-doença, valorizando o cuidado contínuo, com ênfase em atividades de promoção e prevenção e destaque para a orientação familiar. Além disso, a grande maioria das doenças prevalentes na infância exigem medidas simples com baixa complexidade tecnológica e, em casos mais complexos, a APS realiza a coordenação do cuidado, fazendo a ligação com outros níveis de atenção à saúde quando necessário (DAMASCENO SS, et al., 2016). A avaliação é um dos principais pontos para se definir a qualidade de um serviço e consiste num importante instrumento de mudança uma vez que norteia diretrizes para um plano de ação, visando a melhoria da assistência prestada. Existem várias formas de se avaliar a APS, uma delas é o Instrumento de Avaliação da Atenção Primária à Saúde (PCA-Tool/ Primary Care Assessment Tool), que já é validado em vários países e possui versões adaptadas tanto para crianças como para maiores de 18 anos. A versão criança do PCAToolBrasil deve ser direcionado aos pais das crianças ou cuidadores destas (PIMENTEL A, et al., 2019).

O estado do Tocantins destaca-se por possuir alta cobertura de atenção básica do Brasil, mesmo assim, ainda apresenta alto índice de internações por causas sensíveis à atenção básica e baixa cobertura do calendário vacinal de rotina. Com base nisso, há uma constante busca pelo fortalecimento da Atenção Básica no Estado com necessidade de qualificação, apoio institucional, monitoramento e avaliação da estratégia, possibilitando condições para um acompanhamento de qualidade da saúde da população (BRASIL, 2018). Entre os 34 Centros de Saúde e Comunidade (CSC) urbanos de Palmas- TO, o CSC 403 norte foi território da presente pesquisa. Segundo o banco de dados do ESUS - gestor, tem população total adscrita de 10.952 pessoas, sendo aproximadamente 16\% população infantil (menor de 10 anos), em que 196 possuem de 0 a 2 anos.

É necessário priorizar a saúde infantil uma vez que as condições mais prevalentes na infância podem ser resolvidas com tecnologias leves. Assim, o presente trabalho visa avaliar a qualidade da atenção à saúde infantil do ponto de vista dos usuários.

\section{MÉTODOS}

Trata-se de um estudo transversal, de abordagem quantitativa apresentado de acordo com as orientações do STROBE statement (MALTA M, et al., 2010). As variáveis de exposição foram características sócio demográficas e a variável de desfecho foi a qualidade da atenção primária visão dos usuários. A cidade de Palmas foi criada em 1989 e planejada para ser a capital do estado do Tocantins, com população estimada de 299.177 pessoas em 2019 pelo IBGE (BRASIL, 2018).

Teve o sistema de saúde estabelecido, de fato, a partir de 1997 como uma tentativa de organizar o sistema de atenção à saúde para atender às condições da população, orientado no princípio da regionalização, visando a efetividade, eficiência, segurança, qualidade e equidade (SANTANA JP, 2008).

Nesse sentido foi dado ênfase na Saúde da Família em Palmas, como estratégia da atenção básica e como porta de entrada dos serviços. O crescimento acelerado da cidade, com consequente demanda por empregos na área da saúde, favoreceu a estruturação do modelo de atenção básica (GOVERNO DO TOCANTINS, 2015).

O estudo foi realizado em um CSC do Território Kanela, de janeiro a dezembro de 2019. Os dados foram coletados no CSC 403 norte, em consultas, sala de espera, sala de vacina, visitas domiciliares. Os entrevistadores foram previamente apresentados ao modelo de questionário e treinados para realizar a pesquisa. Em setembro de 2019 o CSC 403 norte aderiu ao programa "Saúde na Hora", lançado em maio do mesmo ano pelo governo brasileiro com a proposta de horário de funcionamento estendido. Para incentivar a adesão ao Programa, o Ministério da Saúde aumentou os repasses mensais para as equipes que aderiram (MINISTÉRIO DA SAÚDE, 2019).

Participaram da pesquisa pais ou cuidadores de crianças de 0 a 2 anos que estavam cadastradas no CSC 403 norte. A população do estudo foi composta por crianças de 0 a 2 anos adscritas no CSC, pelas informações 
do E-SUS gestor, com total de 196 crianças. Na pesquisa foi definida o tamanho de amostra de 131 crianças a partir do cálculo amostral, considerando-se um erro amostral de $5 \%$ e nível de confiança de $95 \%$ e admitindo-se uma prevalência de $50 \%$ do evento estudado resultando. Foram excluídos pais ou responsáveis de crianças que tinham mais de 2 anos e também aqueles que não quiseram participar da pesquisa.

O desfecho analisado foi à qualidade da APS prestada às crianças, pela visão dos usuários. Os dados socioeconômico-demográficos foram obtidos por meio da aplicação do questionário com as seguintes variáveis independentes: sexo; idade; número de filhos; estado civil; vínculo empregatício; renda; tempo de atuação; repouso e locais de trabalho.

O instrumento original do PCATool versão criança está disponível na versão Brasil, constituída por 56 itens. Esses itens estão distribuídos nos quatro atributos essenciais da APS, permitindo avaliar os atributos acesso de primeiro contato, longitudinalidade, coordenação dos serviços e a integralidade dos serviços disponíveis e prestados. Os três atributos derivados da APS também são contemplados, possibilitando a avaliação da orientação familiar, orientação comunitária e competência cultural. Além disso, outras três perguntas medem o grau de afiliação ao serviço de saúde (BRASIL, 2011).

As respostas são do tipo Likert, com intervalo de um a quatro para cada atributo. As respostas possíveis para cada um dos itens foram: "com certeza sim" (valor=4), "provavelmente sim" (valor=3), "provavelmente não" (valor=2), "com certeza não" (valor=1) e "não sei / não lembro" (valor=9) (LEÃO CDA, 2011).O grau de avaliação pelo usuário sobre o serviço ofertado foi calculado mediante a utilização do algoritmo estruturados de acordo com as diferentes possibilidades de respostas. Para diminuir o viés de informação, houve treinamento prévio dos internos do curso de Medicina que ajudaram na aplicação dos questionários e foi também explicado ao usuário como funcionaria a pesquisa.

Após a consolidação dos dados relativos a cada atributo, os valores foram transformados em uma escala contínua, variando entre zero e dez. Valores de escores $\geq 6,6$ foram definidos como elevados e equivalentes ao valor três ou mais na escala Likert, indicando forte orientação para a APS. Valores com escores menores $(<6,6)$ foram definidos como baixos indicando fraca orientação para os atributos na APS (LEÃO CDA, 2011). As variáveis qualitativas foram apresentadas por frequências absolutas $(\mathrm{N})$ e frequências relativa (\%). As variáveis quantitativas foram descritas por médias e desvios-padrão, valores mínimos e máximos. O software utilizado foi o Stata® (StataCorp, IC) Versão 11.0.

A participação foi voluntária após assinatura do termo de consentimento informado dos responsáveis. Esse estudo faz parte do projeto guarda-chuva: "Qualidade da Atenção Primária à saúde prestada à população de Palmas Tocantins" e foi aprovado pelo comitê de ética (CAAE: 60080816.4.0000.5516) conforme diretrizes éticas expostas pela Resolução CNS no 466/12 que normatiza pesquisa envolvendo seres humanos.

\section{RESULTADOS}

Dentre os 196 usuários elegíveis para o critério de inclusão, foram 131 participantes ( $n=50 \%)$. Dentre os 196 usuários elegíveis para o critério de inclusão, 131 participaram, com predominância do sexo feminino ( $n=96 ; 90,57 \%)$ e idade média de 28,7 (dp $\pm 6,6)$. Em relação à renda mensal média tivemos resultado de 1568,11 ( $d p \pm 811,8)$ A maior parte da amostra tem 2 filhos $(n=42 ; 42,98 \%)$, seguido de 3 ou mais filhos $(n=35 ; 30,7 \%)$. Outra característica que pode ser observada entre os entrevistados é que a maioria tem companheiro $(n=96 ; 84,21 \%)$. Em relação à ocupação grande parte dos que responderam esse item é vinculado por meio de contrato $(n=39 ; 95,12 \%)$ e com carga horária de trabalho semanal alta com de mais de 12horas/semana $(n=40$; $93,02 \%)$. O tempo de serviço na instituição entre 1 e 5 anos apareceu entre a maioria dos entrevistados ( $n=22$; $53,66 \%)$ bem como pouco tempo de repouso, sendo 1 hora por dia $(n=20 ; 40,78 \%)$. A Tabela 1 consolida as principais características sócio econômicas demográficas dos cuidadores de crianças. 
Tabela 1 - Aspectos socioeconômicos demográficos dos(as) cuidadores(as) de crianças usuárias da APS em Palmas/TO, 2019.

\begin{tabular}{|c|c|c|}
\hline Variáveis & $\mathbf{N}$ & $\%$ \\
\hline \multicolumn{3}{|l|}{ Sexo } \\
\hline Masculino & 10 & 9,43 \\
\hline Feminino & 96 & 90,57 \\
\hline \multicolumn{3}{|l|}{ Número de filhos } \\
\hline 1 filho & 35 & 30,7 \\
\hline 2 filhos & 49 & 42,98 \\
\hline 3 ou mais filhos & 30 & 26,32 \\
\hline \multicolumn{3}{|l|}{ Companheiro } \\
\hline Não & 18 & 15,79 \\
\hline Sim & 96 & 84,21 \\
\hline \multicolumn{3}{|c|}{ Vínculo empregatício } \\
\hline Concurso & 2 & 4,88 \\
\hline Contrato & 39 & 95,12 \\
\hline \multicolumn{3}{|l|}{ Carga horária } \\
\hline 6 a 8 horas & 1 & 2,33 \\
\hline 10 a 12 horas & 2 & 4,65 \\
\hline Mais de 12 horas & 40 & 93,02 \\
\hline \multicolumn{3}{|l|}{ Tempo de serviço } \\
\hline Menos de 1 ano & 8 & 19,51 \\
\hline 1 a 5 anos & 22 & 53,66 \\
\hline 6 a 10 anos & 10 & 24,39 \\
\hline 11 a 19 anos & 1 & 2,44 \\
\hline \multicolumn{3}{|l|}{ Repouso } \\
\hline Sem descanso & 5 & 12,2 \\
\hline 1 hora & 20 & 48,78 \\
\hline 2 horas & 14 & 34,15 \\
\hline Locais de trabalho & 2 & 4,88 \\
\hline 1 local & 38 & 100 \\
\hline 2 locais & - & - \\
\hline 3 locais & - & - \\
\hline \multirow{2}{*}{ Idade } & Média(dp) & Min.; Máx. \\
\hline & $28,7(6,6)$ & $15 ; 47$ \\
\hline Renda (R\$) & $1568,11(811,8)$ & $100 ; 5000$ \\
\hline
\end{tabular}

Legenda: Dp: Desvio-padrão; Min.: Valor mínimo; Máx.: Valor Máximo; R\$: Reais.

Fonte: Nunes TRB, et al., 2020.

Em relação a qualidade da APS, os escores médios para atributos são apresentados baseados na percepção dos usuários da atenção primária à saúde no CSC 403 norte em Palmas/Tocantins (Tabela 2). 
Tabela 2 - Atributos da atenção primária avaliada pelo PCATool.

\begin{tabular}{lcccc}
\hline Atributos & Média & Desvio padrão & Mínimo & Máximo \\
\hline Acesso de Primeiro Contato - Afiliação & 10 & 0 & 10 & 10 \\
Acesso de Primeiro Contato - Utilização & 8,4 & 1,7 & 3,3 & 10 \\
Acesso de Primeiro Contato - Acessibilidade & 4,9 & 2,2 & 0 & 8,9 \\
Longitudinalidade & 6,6 & 1,4 & 1,7 & 9,5 \\
Coordenação - Integração de Cuidados & 7,4 & 3,8 & 0 & 10 \\
Coordenação - Sistema de Informações & 8,4 & 1,7 & 3,3 & 10 \\
Integralidade - Serviços Disponíveis & 8,0 & 1,7 & 2,2 & 10 \\
Integralidade - Serviços prestados & 7,7 & 2,8 & 0 & 10 \\
Orientação Familiar & 7,0 & 2,5 & 0 & 10 \\
Orientação Comunitária & 5,8 & 2,6 & 0 & 10 \\
\hline
\end{tabular}

Fonte: Nunes TRB, et al., 2020.

Os atributos essenciais apresentados com maior média foram: Afiliação [10 (dp 0,0)], Acesso de Primeiro Contato - Utilização [8,4 (dp $\pm 1,7)]$, Coordenação - Sistema de Informação [8,21 (dp $\pm 1,7)]$ e Integralidade Serviços Disponíveis [8,0 (dp $\pm 1,7)]$. A longitudinalidade apresentou escore no limite esperado $[6,6(\mathrm{dp} \pm 1,4)]$.

Por outro lado, os menores escores foram atribuídos à acessibilidade [4,9 (dp $\pm 2,2)]$ e à orientação comunitária [5,8 (dp $\pm 2,6)]$. A orientação familiar $[7,0(\mathrm{dp} \pm 2,5)]$ foi avaliada com escore pouco acima do ponto de corte. Ao analisar as medianas dos escores Essencial e Geral do PCATool na população estudada, observa-se a partir da figura 1 que a mediana do Escore Essencial foi de aproximadamente 6,8 e do Escore Geral de 5,8 (Figura 1).

Figura 1 - Média dos Escores Essencial e Geral.

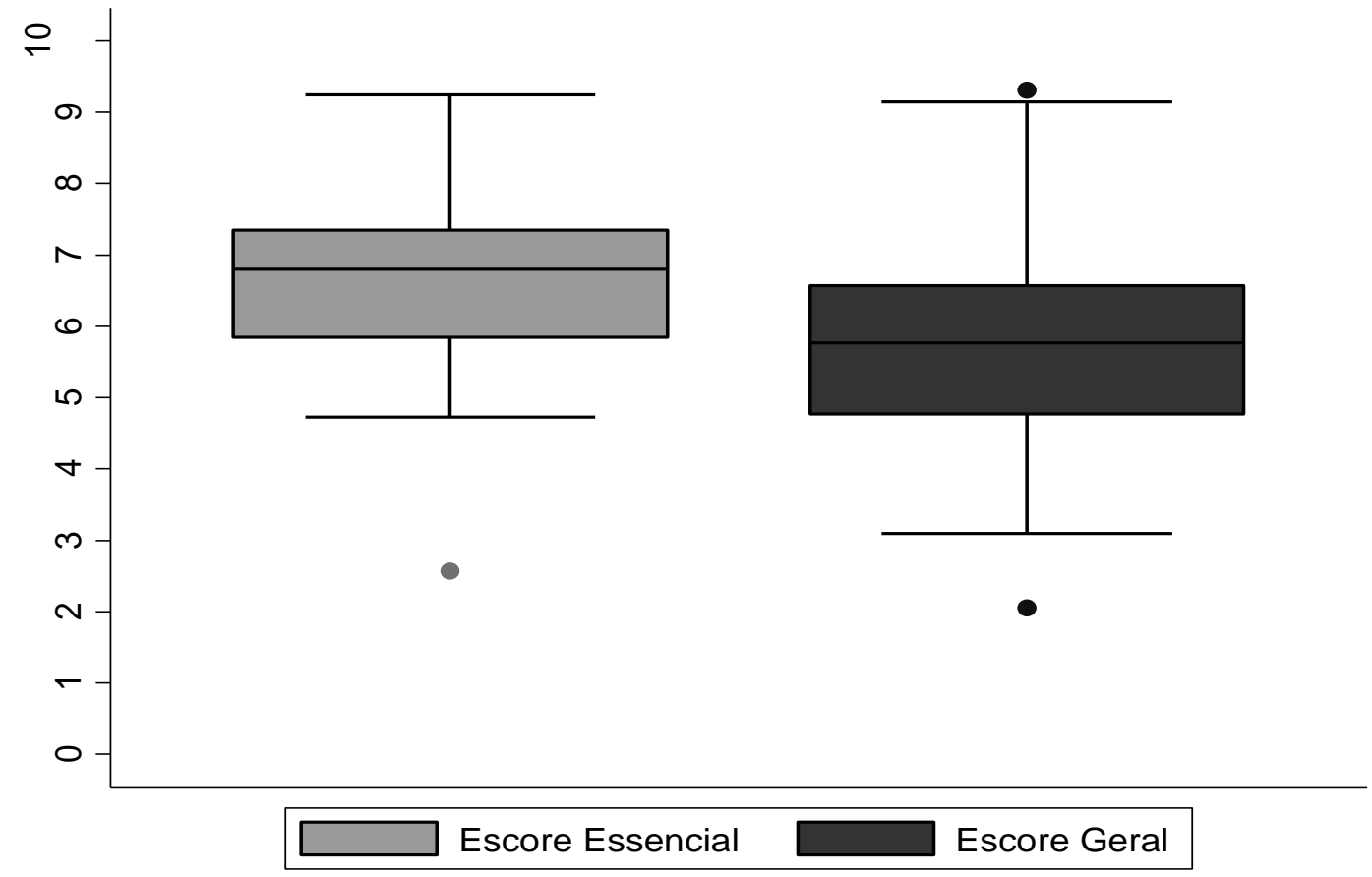

Fonte: Nunes TRB, et al., 2020. 


\section{DISCUSSÃO}

No presente artigo, descrevemos do ponto de vista dos usuários a qualidade do atendimento dos serviços de saúde em relação à saúde infantil. Os principais itens que se destacaram foram a integralidade e coordenação do cuidado, com altos escores indicando forte orientação para os atributos da APS. Em contrapartida, com baixos escores apareceram a acessibilidade e a orientação comunitária, representando desafios a serem trabalhados a fim de melhorar a assistência primária à saúde.

De acordo com a caracterização da amostra, observa-se que a maioria dos usuários entrevistados era do gênero feminino, com média de 2 filhos ou mais, vínculo empregatício mediante contrato e renda mensal média de 1568,11 reais. A alta carga horária de trabalho semanal também foi observada na caracterização dos entrevistados, sendo de mais de 12 horas semanais.

Uma renda mensal baixa associada à quantidade de filhos pode se relacionar a maior dependência do sistema público de saúde. Segundo Santos JP (2012) na teoria da casualidade entre renda e saúde é esperado que pessoas que tenham menores condições financeiras adoeçam mais devido a condições de moradia, saneamento, acesso a medidas preventivas. Isso acontece devido a vulnerabilidade maior a doenças, principalmente em crianças.

No âmbito da saúde da criança, os serviços orientados à APS são capazes de reduzir as internações hospitalares uma vez que os principais problemas relacionados às crianças são condições sensíveis à atenção primária como afecções respiratórios e gastroenterites (DAMASCENO SS, et al.,2016).

A maioria dos atributos da APS recebeu avaliação considerada satisfatória de acordo com os escores calculados. O maior escore foi o de grau de afiliação com o Serviço de Saúde [10 (dp 0,0)] e relaciona-se ao grau de identificação do usuário com o serviço de saúde, ou seja, o quanto o usuário reconhece o serviço ou o profissional de saúde como referência para a assistência à saúde da criança (LEÃO CDA, 2011). A análise da afiliação objetiva identificar o serviço ou profissional de saúde que serve como referência para o usuário em questão (BRASIL,2010).

Tal avaliação com altos escores é indicativo de um bom vínculo com a unidade de saúde, característica que fortalece a APS, pois o usuário a vê como fonte de cuidados. O atributo essencial acesso de primeiro contato é formado por dois componentes: acessibilidade e utilização. Na avaliação desses componentes, foi observado uma divergência sendo que o componente utilização $[8,4(\mathrm{dp} \pm 1,7)]$ teve altos escores enquanto a acessibilidade [4,9 (dp $\pm 2,2)]$ foi avaliada com baixa pontuação, enfraquecendo a APS. A acessibilidade refere-se às características que facilitam ou dificultam a chegada das pessoas aos serviços, podendo ser sócio organizacionais, financeiras (características do serviço) e geográficas (relacionadas a distância, tempo e custo de viagem entre outros.

Conforme a Política Nacional de Atenção Básica (PNAB) os serviços de atenção primária são a principal porta de entrada do sistema de saúde no Brasil e espera-se que seja acessível e resolutivo em relação às necessidades de saúde. Tal acessibilidade pode ser fazer através de escuta qualificada no acolhimento, demanda espontânea, horário de atendimento estendido e o primeiro atendimento às urgências (KESSLER M, et al., 2018). Logo, o baixo valor atribuído ao item acessibilidade indica fragilidade nesse ponto no Centro de Saúde em questão, item esse que precisa ser melhorado pois funciona como barreira para o contato do usuário com a unidade e prejudicando todos os demais itens que a APS deve ter para se consolidar.

A adesão do CSC 403 norte ao programa "Saúde na Hora" já caracteriza uma tentativa de melhorar a acessibilidade previamente vista como deficitária pelos gestores. Desde setembro de 2019, o Centro de Saúde funciona em horário diferenciado da maioria ( $7 \mathrm{~h}$ às $19 \mathrm{~h})$ no intuito de aumentar a acessibilidade. Em relação à utilização, também componente do primeiro contato, os escores médios foram altos, significando que o usuário procura o Centro de Saúde regularmente a cada vez que necessita de atenção em caso de doença ou acompanhamento rotineiro, isso potencializa os vínculos e o cuidado continuado (KESSLER M, et al., 2018). 
A longitudinalidade apresentou escore no limite esperado $[6,6(\mathrm{dp} \pm 1,4)]$ demonstrando fragilidade dos serviços uma vez que se refere à continuidade da assistência e à qualidade da relação profissional-paciente (KESSLER M, et al., 2018). Entre os atributos essenciais, a longitudinalidade se destaca por compreender 0 vínculo do usuário com a unidade e/ou com o profissional. A Unidade deve ser fonte regular de atenção à saúde e o profissional deve conhecer e se responsabilizar pelo atendimento destes indivíduos, levando em conta a corresponsabilização do cuidado (KESSLER M, et al., 2018).

Um dos pontos que pode prejudicar o atributo longitudinalidade é a alta rotatividade de profissionais, principalmente médicos, no Centro de Saúde avaliado, pois, entre outras questões, é uma unidade que recebe residentes de Medicina de Família e Comunidade que cumprem suas funções durante o período de 2 anos e depois não pertencem, necessariamente, àquela área em que se formou. $A$ integralidade abrange serviços disponíveis e serviços prestados, objetiva reconhecer a heterogeneidade das necessidades do usuário levando em conta características biopsicossociais e oferecer serviços em diversos âmbitos desde a prevenção, cura até a reabilitação (KESSLER M, et al., 2018).

Serviços Disponíveis $[8,0(\mathrm{dp} \pm 1,7)]$ referem-se à experiência do usuário com o serviço de saúde e o conhecimento que ele tem do que é oferecido pelo Centro de Saúde como serviço (BRASIL, 2011). Serviços Prestados 7,7 [(dp $\pm 2,8)]$ refere-se ao que é abordado durante os atendimentos. A integralidade da atenção primária consiste em produzir saúde focado na pessoa e não na doença em si, analisando os aspectos que estão envolvidos além do aspecto orgânico (CARNUT L, 2017).

Em relação aos itens Integração de Cuidados [7,4 (dp $\pm 3,8)]$ e Sistema de Informação $[8,21$ (dp $\pm 1,7)]$ as avaliações foram satisfatórias. Esses itens compõem o atributo essencial coordenação do cuidado, que integra serviços relacionados à saúde, em qualquer complexidade que o usuário tenha sido atendido e ocorre por meio da identificação de informações de consultas prévias e de consultas em outro nível de atenção à saúde. Dentre os fatores que podem ter contribuído para a boa avaliação nesse quesito destaca-se a existência do prontuário eletrônico do cidadão (PEC) dentro do sistema de atendimento na rede de atenção à saúde em Palmas -TO. Todas as consultas realizadas pelo SUS são registradas no sistema E-SUS o que permite acompanhamento do prontuário do paciente, facilitando a coordenação do cuidado.

Como atributo derivado aparece a Orientação familiar $[7,0(\mathrm{dp} \pm 2,5)]$ com avaliação pouco acima do ponto de corte, porém satisfatória. Nesse item é levado em conta o quanto os usuários participam das decisões de seu tratamento ou de seus familiares (BRASIL, 2010). São considerados contexto familiar, conhecimento dos membros da família, dinâmica familiar, doenças existentes na família (KESSLER M, et al., 2018).

De Acordo com a OMS (2008), no Relatório Mundial da Saúde, o "Foco nas pessoas e famílias" reflete melhora na promoção/prevenção em saúde além de maior compreensão de aspectos psicológicos das pessoas bem como aumento do vínculo e adesão ao tratamento. O enfoque familiar também influi na melhoria da qualidade de vida, maior comunicação e confiança dos usuários nos profissionais. Por isso a importância de investir cada vez mais na família como unidade de cuidado.

O atributo derivado orientação comunitária $[5,8(\mathrm{dp} \pm 2,6)]$ objetiva envolver a comunidade em integração com o Conselho local de Saúde e o CSC. (BRASIL, 2011). Apresentou escore abaixo do esperado, demonstrando fragilidade dos serviços em relação conhecimento da população adscrita e da participação popular. Esse atributo está relacionado ao conhecimento das necessidades da população cadastrada no território, conhecimento dos problemas mais prevalentes na área e dos recursos disponíveis na comunidade (KESSLER M, et al., 2018).

Uma alternativa para melhorar a orientação comunitária da APS é reforçar a representação da comunidade nas decisões sobre saúde por meio do Conselho local de saúde, para que assim a população se veja como parte efetiva e ativa do CSC e não apenas como usuária. Outro ponto a ser fortalecido é o diagnóstico situacional da área e a intensificação das pesquisas durante visita domiciliar de acordo com a epidemiologia local. Baseado no conhecimento da epidemiologia local é que são propostas as ações de prevenção, promoção e educação em saúde. 
A pesquisa foi realizada em apenas um CSC de Palmas o que pode ser considerado um fator limitante do estudo já que existem cerca de 34 Centros de Saúde distribuídos em vários territórios de Palmas - TO. Outro ponto limitante é a heterogeneidade da população que faz com que alguns utilizem mais outros menos os serviços disponibilizados, de acordo com a necessidade. A análise dos resultados da pesquisa mostra a importância de se atentar para pontos fundamentais da APS que estão enfraquecidos como a acessibilidade e a orientação comunitária. Trata-se de um desafio a ser encarado por todos os profissionais para fortalecer a atenção básica em saúde. A cidade de Palmas -TO tem um enorme potencial uma vez que já possui uma estrutura de Atenção Primária bem definida e está em pleno desenvolvimento, sendo um ótimo campo para aprimorar pontos falhos.

\section{CONCLUSÃO}

Os usuários têm uma visão satisfatória dos serviços de saúde infantil como um todo, já que a maioria dos atributos obteve boa avaliação, representando forte orientação para a APS. Porém, alguns pontos importantes como acessibilidade e orientação comunitária tiverem avaliação baixa, implicando em dificuldade na utilização e comprometimento da qualidade dos serviços. Tais fatores precisam ser melhorados pois a APS é tida como componente fundamental dos sistemas de saúde sendo ponto de início da atenção à saúde e filtro para acesso aos serviços especializados e todos os atributos estão relacionados entre si, potencializando uns aos outros. Dessa forma, entende-se que além a contribuição científica esse estudo apresenta um caráter social e de reestruturação dos serviços ofertados a fim de auxiliar no desenvolvimento de políticas públicas no setor da gestão e assistência.

\section{REFERÊNCIAS}

1. ALMEIDA PFDE, et al. Coordenação do cuidado e Atenção Primária à Saúde no Sistema Único de Saúde. Saúde em Debate, 2018; 42(1): 244-260.

2. BRANQUINHO ID, LANZA FM. Saúde da Criança na Atenção Primária: Evolução das Políticas Brasileiras e a Atuação do Enfermeiro. 2018

3. BRASIL. Secretaria de Saúde do Estado do Tocantins, 2018

4. BRASIL. Ministério da Saúde. Secretaria de Atenção em Saúde. Departamento de Atenção Básica. Manual do instrumento de avaliação da atenção primária à saúde: primary care assessment tool pcatool - Brasil / Ministério da Saúde, Secretaria de Atenção em Saúde, Departamento de Atenção Básica. - Brasília: Ministério da Saúde, 2011.

5. CARNUT L. Cuidado, integralidade e atenção primária: articulação essencial para refletir sobre o setor saúde no Brasil. Saúde em Debate, 2017; 41(115): 1177-1186.

6. DAMASCENO SS, et al. Saúde da criança no Brasil: Orientação da rede básica à atenção primária à saúde. Ciencia e Saude Coletiva, 2016; 21(9): 2961-2973.

7. FREITAS TCC, et al. A Atenção Primária como parte integrante da rede de atendimento as Urgências e Emergências: à luz da literatura. Revista Eletrônica Acervo e Saúde, 2020; (38).

8. GIOVANELLA L, et al. De Alma-Ata a Astana. Atenção primária à saúde e sistemas universais de saúde: compromisso indissociável e direito humano fundamental. Cadernos de saude publica, 2019; 35(3): e00012219.

9. GOVERNO DO ESTADO DO TOCANTINS SECRETARIA DE ESTADO DA SAÚDE. Regionalização da Saúde no Estado do Tocantins: História e Mapas, 2015.

10. KESSLER M, et al. A longitudinalidade na Atenção Primária à Saúde: comparação entre modelos assistenciais. Rev Bras Enfermagem, 2018; 71(3): 1127-1135.

11. LEÃO CDA, et al. Atributos da atenção primária na assistência à saúde da criança: avaliação dos cuidadores. Revista Brasileira de Saúde Materno Infantil, 2011.

12. MALTA M, et al. STROBE initiative: guidelines on reporting observational studies. Revista de Saude Publica, 2010; 44(3): 559565.

13. MINISTÉRIO DA SAÚDE, Brasil. Programa "Saúde na Hora", Portaria no 2.436/GM/MS, de 2017, a Portaria de Consolidação no 2/GM/MS, de 2017, a Portaria de Consolidação n 6/GM/MS, de 2017, e dá outras providências. Portaria № 930, de 15 de Maio de 2019. Brasília - DF, maio 2019.

14. OMS. Cuidados de Saúde Primários: Agora mais do que nunca, 2008.

15. PEITER CC, et al. Healthcare networks: trends of knowledge development in Brazil. Escola Anna Nery, 2019; 23(1): 1-10.

16. PIMENTEL A, et al. Avaliação dos atributos da Atenção. Primária à Saúde de Fortaleza, 2019; 72(1): 24-31.

17. PORTELA GZ. Atenção Primária à Saúde: um ensaio 255. Physis Revista de Saúde Coletiva, 2016; $27(2): 255-276$.

18. RIBEIRO LCC, et al. Acolhimento às crianças na atenção primária à saúde: um estudo sobre a postura dos profissionais das equipes de saúde da família. Cad. Saúde Pública, 2010; 26(12): 2316-2322.

19. SANTANA JP, et al. Caracterização do processo de trabalho em atenção básica/saúde da família - Aspectos Institucionais, do Emprego e da Atividade do Médico nos Municípios de Campo Grande/MS, Cuiabá/MT, Goiânia/GO e Palmas/TO - Universidade de Brasília, UnB, Brasília - DF, 2008.

20. STARFIELD B. Atenção primária: equilíbrio entre necessidades de saúde, serviços e tecnologia / Primary care: balancing health needs, services, and technology, 2002. 